\title{
Expression of integrins and extracellular matrix proteins at the maternal-fetal interface during tubal implantation
}

\author{
L. Qin ${ }^{1}$, Y. L. Wang ${ }^{1 *}$, S. X. Bai ${ }^{1}$, Z. J. Xiao ${ }^{1}$, R. Herva ${ }^{2}$ and Y. S. Piao ${ }^{1}$ \\ ${ }^{1}$ State Key Laboratory of Reproductive Biology, Institute of Zoology, Chinese Academy of Sciences, \\ Beijing 100080, China; and ${ }^{2}$ Department of Pathology, University of Oulu Central Hospital,
}

Investigation of the expression pattern of integrins and their extracellular matrix (ECM) ligands in trophoblasts at the maternal-fetal interface during tubal pregnancy may aid better understanding of the adhesion and invasion of acceptable maternal endometrium by trophoblast cells at the very early stage of human gestation. In this study, spatial and temporal alterations of integrins and ECM ligands were examined in specimens of tubal pregnancies during weeks 3-9 of gestation. In situ hybridization and immunohistochemistry revealed that relatively high levels of integrin $\alpha_{1}, \beta_{1}, \alpha_{5}$ subunits and heterdimer $\alpha_{5} \beta_{1}$ as well as ECM ligands, were displayed in trophoblast cells as early as weeks 3-4 of gestation. Expression peaked during weeks 5-7 and then, with the exception of integrin $\alpha_{1}$, which remained high, declined slightly up to weeks 8-9 of gestation. Immunoreactive fibronectin, laminin and type IV collagen were detected in column cytotrophoblastic cells (CTB) and some invasive extravillous cytotrophoblast (EVCT) cells and the alterations were coincident with those of the corresponding integrin receptors in EVCT cells. Laminin was strongly stained in EVCT cells that had invaded maternal blood vessels and deep into the interstitium. Maternal epithelial, endothelial and stromal cells also expressed these integrins and ECM ligands. The results indicate their involvement in mediating the adhesion of trophoblasts to the epithelium of the maternal Fallopian tube. The upregulated expression of these molecules in column CTB and invasive EVCT cells may also facilitate the invasion of trophoblasts into the maternal interstitium. Moreover, trophoblasts possessed the potential for selfcontrolled adhesion and invasion and appear to reach peak invasive capability in the second month of tubal implantation.

\section{Introduction}

Implantation is introduced by the attachment of blastocystic trophoblasts to acceptable uterine epithelium during a special stage of pregnancy known as the implantation window. It is generally agreed that the adhesion and invasion of the uterine endometrium by trophoblast cells are crucial events during embryo implantation. This complex process involves the coordinated interaction of adhesive molecules, extracellular matrix (ECM) ligands, matrix metalloproteinases (MMPs) and tissue inhibitors of MMPs (TIMPs) (Fisher and Damsky, 1993; Huppertz et al., 1998). ECM ligands regulate cell adhesion, migration, differentiation and signal transduction by binding to the corresponding integrin receptors (Duband, 1990; Lin and Bissell, 1993; Wallner et al., 1998). Usually fibronectin targets integrin $\alpha_{5} \beta_{1}$ and $\alpha_{3} \beta_{1}$, whereas laminin interacts with $\alpha_{6} \beta_{4}, \alpha_{1} \beta_{1}$ and $\alpha_{3} \beta_{1}$, and type IV collagen (Col IV) binds to $\alpha_{1} \beta_{1}$ and $\alpha_{2} \beta_{1}$ (Rubin et al., 1996). Integrin links with ECM ligands

*Correspondence

Email: wangyl@panda.ioz.ac.cn through recognition of the Arg-Gly-Asp (RGD) peptides, leading to the activation of focal adhesion kinase and thus triggering the early step of the signal transduction cascade (Schaller et al., 1992; Schoenwaelder and Burridge, 1999; Critchley, 2000).

The roles of integrins and their corresponding ECM ligands in embryo implantation and placentation have been studied mainly in gene knockout mice. Evidence has indicated that mice with a deleted integrin $\alpha_{1}$ gene underwent normal development, but developed a specific deficit in cell adhesion (Gardner et al., 1996). On the other hand, mice with a homozygous null mutation in the $\alpha_{5}$ gene displayed defects in posterior trunk and yolk sac mesodermal structures by day 9 of gestation and died at day 10-11 (Yang et al., 1993). $\beta_{1}$-gene mutant mice died early after implantation (Stephens et al., 1995). Careful examination of implantation sites indicated that interaction between trophoblast $\beta_{1}$ integrin and maternal ECM ligands might be important for placentation. Meanwhile, mice with a homozygous null mutation of the fibronectin gene died at the embryonic stage (Watt and Hodivala, 1994). Laminin and Col IV, important constituents of the basement membrane, are 
widely distributed in various tissues (Earl et al., 1990; Van der Rest and Garrone, 1991; Virtanen et al., 2000). Targeted deletion of the LAMC1 gene encoding the $\gamma_{1}$ subunit of laminin caused failure in formation of the basement membrane, which led to earlier embryonic lethality (Smyth et al., 1998). Col IV has also been postulated to play an important role in the maintenance of pregnancy (Furuhashi et al., 1994).

Over the past decades, considerable effort has been made towards understanding the mechanism controlling embryo implantation in humans. It has been disclosed that the interaction of fibronectin with integrin $\alpha_{5} \beta_{1}$ significantly contributes to the anchorage of placental cells to uterine ECM, whereas integrin $\alpha_{1}$ is involved in the subsequent process of placentation (Aplin et al., 1999; Tarone et al., 2000). In addition, there has been evidence indicating that interaction between $\alpha_{1} \beta_{1}$ and laminin or Col IV can promote invasion of trophoblast cells in vitro (Damsky et al., 1994). However, owing to the limited availability of human specimens containing the intact maternal-fetal interface of normal pregnancy, much remains unclear concerning the expression patterns of the relevant molecules involved in the chemical dialogue between trophoblasts and the maternal endometrium, especially at the very early stage of embryo implantation.

Tubal pregnancy is a type of abnormal gestation, in which an embryo implants in the Fallopian tube rather than the uterus. However, there is evidence to indicate that tubal pregnancy triggers normal immunoreaction and hormonal activation of the maternal body (Earl et al., 1986). Some implantation-related molecules, such as MMPs, TIMPs, urokinase plasminogen activator and its receptor show similar patterns of expression in trophoblast cells during tubal pregnancy as those in normal uterine pregnancy (Floridon, 1999; Kucera et al., 2000). Furthermore, fetal-Fallopian samples removed from women with tubal pregnancies often possess embryos with normal morphology and an intact maternalfetal interface. It is even possible to obtain such samples at very early stages of gestation. Therefore, tubal pregnancy provides a suitable analogue for the investigation of the mechanism of trophoblast adhesion and invasion during the very early stages of implantation.

In the present study, expression patterns and dynamic changes of the integrin $\alpha_{1}, \alpha_{5}, \beta_{1}$ subunits and heterodimer $\alpha_{5} \beta_{1}$ as well as their ECM ligands including fibronectin, laminin and Col IV at the maternal-fetal interface were studied by immunohistochemistry and in situ hybridization in human specimens collected from week 3 to week 9 of tubal pregnancy.

\section{Materials and Methods}

\section{Tissue specimens}

With the permission of the Local Ethical Committee, 23 histological normal specimens from tubal pregnancies at weeks 3-9 of gestation were selected from hundreds of the tissue archives between 1997-2000 at the Department of Pathology, Oulu University Hospital (Oulu, Finland). The hospital has kept the whole clinic records for all the archive materials that were kept in appropriate conditions. In the selected 23 cases, there were the intact implantation sites, and the Fallopian was unruptured according to the clinic records. The gestational age of specimens was finally determined based on the morphological markers (development of the primary, secondary and tertiary villi, appearance of fetal red blood cells, and development of villous capillary) widely accepted in uterine pregnancy (Kaplan, 1999). At least three representative specimens of each stage (week) of gestational development were collected from the available archived material. Specimens were fixed in $4 \%$ paraformaldehyde (PFA) at $4{ }^{\circ} \mathrm{C}$ overnight, dehydrated routinely and embedded in paraffin wax (solidification point $51-53^{\circ} \mathrm{C}$ ). Six $\mu \mathrm{m}$ sections were prepared in Mocrotome RM2135 (Leica) and collected on Super Frost+ glass slides (Menzel-Gläser). The sections were stained with haematoxylin and eosin for pathological diagnosis. In addition, immunostaining of cytokeratin was performed for the identification of trophoblast cells.

\section{Immunohistochemistry}

Staining for a certain antibody was performed in one batch with sections consisting of every specimen at various gestational ages. All the conditions were kept strictly the same for every section. Each experiment was repeated at least three times and similar results were obtained. In detail, sections were deparaffinized, rehydrated and then retrieved in $10 \mathrm{mmol}$ citrate buffer $\mathrm{I}^{-1}(\mathrm{pH} 6.0)$ at $95^{\circ} \mathrm{C}$ for $15 \mathrm{~min}$. After treatment with $1 \%$ hydrogen peroxide for $15 \mathrm{~min}$, sections were incubated with unique primary antibody diluted with TBS containing $1 \%$ bovine serum albumin at $4^{\circ} \mathrm{C}$ overnight. Antibodies used in the study were: rabbit polyclonal antibodies against fibronectin (F-3648, 1:500) and laminin (L-9393, 1:200) (Sigma International, St Louis, $\mathrm{MO})$, mouse monoclonal antibodies to Col IV (MAB $1910,1: 500$ ), integrin $\beta_{1}$ subunit (MAB 1951, 1:500), integrin $\alpha_{5} \beta_{1}$ (MAB 1969, 1:100), and rabbit polyclonal antibody against integrin $\alpha_{1}$ (AB 1934, 1:200) (Chemicon International, Temecula, CA). The negative control was assessed with non-immune serum for the polyclonal antibodies. Pre-absorbed antibodies with purified human Col IV (12168-019) (Life Technologies Inc., Rockville, MD) or integrin $\alpha_{5} \beta_{1}$ (CC1026; Chemicon) were used as negative controls for the corresponding monoclonal antibodies. Final visualization was achieved using the Dako Envision ${ }^{\mathrm{TM}}$ System (Dako Corporation, Carpinteria, CA). Counterstaining with haematoxylin was performed before the slides were mounted. The results were then evaluated by three observers who did not know the gestational age of samples. 
Table 1. Primer sets used for the preparation of in situ hybridization probes

\begin{tabular}{lllc}
\hline Gene & Upper primer sequences $\left(5^{\prime} \rightarrow 3^{\prime}\right)$ & Lower primer sequences $\left(5^{\prime} \rightarrow 3^{\prime}\right)$ & Product length $(\mathrm{bp})$ \\
\hline Integrin $\alpha_{1}$ & TGGTTATTGTGACAGATGG & ATAGGCTCCTACTGCTCC & 371 \\
Integrin $\alpha_{5}$ & AAGCCTGAGGCAGTGCTATTCC & CGCAGCCTGAAACACTCAGC & 365 \\
Integrin $\beta_{1}$ & AGTACTTGTGAAGCCAGCAACG & TGGGACACTCTGGATTCTCC & 371 \\
\hline
\end{tabular}

\section{Preparation of digoxigenin-labelled probes}

Digoxigenin (DIG)-labelled cRNA probes were used for in situ hybridization. Briefly, human chorionic villi tissues were obtained from patients who had had therapeutic termination of pregnancy in weeks 6-7 of gestation (Haidian Hospital, Beijing). The informed consent of all patients was obtained. Total RNA was isolated from these tissues with TRIzol reagent (Life Technologies Inc., Grand Island, NY). The cDNA fragments of human integrin $\alpha_{1}, \beta_{1}$ and $\alpha_{5}$ were obtained by RT-PCR using the primer sets summarized in Table 1. The sequences of the RT-PCR products were verified by DNA sequencing. The fragments were then constructed into PGEM-T easy vector (Promega). The cRNA probes were transcribed in vitro, either by T7 or SP6 RNA polymerase, from the linearized DNA constructs according to the instructions provided with the DIG RNA Labelling System (Roche Diagnostics $\mathrm{GmbH}$, Mannheim). The probes were kept at $-80^{\circ} \mathrm{C}$ at a concentration of $0.1 \mu \mathrm{g} \mu \mathrm{I}^{-1}$.

\section{In situ hybridization}

In situ hybridization was performed as described by Braissant and Wahli (1998). In brief, the sections were routinely deparaffinized and rehydrated. After treatment with $0.2 \mathrm{~mol} \mathrm{HCl} \mathrm{I}^{-1}$ for $15 \mathrm{~min}$, the samples were heatdenatured at $70^{\circ} \mathrm{C}$ for $15 \mathrm{~min}$ in $2 \times \mathrm{SSC}$ and then digested with $4 \mu \mathrm{g} \mathrm{ml}^{-1}$ of proteinase $\mathrm{K}$ (Boehringer Mannheim, Mannheim) at $37^{\circ} \mathrm{C}$ for $15 \mathrm{~min}$. The sections were post-fixed in $4 \%$ PFA at room temperature $\left(20-25^{\circ} \mathrm{C}\right)$ for $10 \mathrm{~min}$ and washed twice with PBS containing $0.1 \%$ active DEPC (Sigma) for $15 \mathrm{~min}$. Acetylation was carried out in triethanolamine buffer containing $0.5 \%$ acetic anhydride for $10 \mathrm{~min}$, after which the specimens were equilibrated in $5 \times$ SSC for 15 min. Prehybridization was performed at $58^{\circ} \mathrm{C}$ for $4 \mathrm{~h}$ in prehybridization buffer $\left(50 \%\right.$ formamide, $20 \mathrm{mmol}$ Tris- $-\mathrm{HCl} \mathrm{I}^{-1}, 50 \mathrm{mmol}$ EDTA I ${ }^{-1}, 0.5 \mathrm{mg}$ tRNA coli $\mathrm{ml}^{-1}, 100 \mathrm{mmol}^{\text {DTT I }}{ }^{-1}$ ), after which sections were further hybridized for $18 \mathrm{~h}$ at $58^{\circ} \mathrm{C}$ in fresh hybridization buffer containing $1 \mathrm{ng}$ cRNA $\mu \mathrm{I}^{-1}$ antisense probes, replacing antisense probes with cRNA sense probes for negative control. After washing in consecutive baths of $2 \times$ SSC and $0.1 \times$ SSC at $65^{\circ} \mathrm{C}$ for $1 \mathrm{~h}$, slides were blocked with $0.5 \%$ blocking reagent (Boehringer Mannheim) and then incubated with alkaline phosphatase-coupled antidigoxigenin antibody (dilution 1:500) for $2 \mathrm{~h}$ at room temperature. Colour development was performed in a buffer composed of $100 \mathrm{mmol}$ Tris- $\mathrm{HCl} \mathrm{I} \mathrm{I}^{-1}, \mathrm{pH}$ 9.5, $100 \mathrm{mmol} \mathrm{NaCl} \mathrm{I-1}, 50 \mathrm{mmol} \mathrm{MgCl}_{2} \mathrm{I}^{-1}, 4.5 \mu \mathrm{l}$ 4-nitro blue tetrazolium chloride and $3.5 \mu \mathrm{l}$ x-phosphate/5bromo-4-chloro-indolyl-phosphate (Boehringer Mannheim). Non-specific staining was removed by rinsing in $95 \%$ ethanol. After dehydration, slides were mounted in resin. The results were assessed based on the evaluation of three observers who did not know the gestational age of samples.

\section{Results}

Temporal and spatial changes of distribution of fibronectin, Col IV and laminin at the maternal-fetal interface during weeks 3-9 of tubal implantation

Immunohistochemical assay showed that the distribution patterns of fibronectin and Col IV at the maternalfetal interface of tubal pregnancy were similar. Signals for both kinds of ECM ligands were displayed in cytoplasm and extracellular regions of the villous mesenchyme, distal column cytotrophoblastic cells (CTB) and invasive extravillous cytotrophoblast (EVCT) cells (Fig. 1a-c,d-f), but were not detected in villous CTB (VCT) and syncytiotrophoblast. The signal intensity increased from distal column CTB to EVCT, but decreased in those EVCT that had invaded deeply into the maternal side (Fig. 2a,b). Concurrently, EVCT cells displayed fewer intracellular signals and more extracellular signals the deeper they penetrated maternal tissue (Fig. 2a,b). The difference in distribution of fibronectin and Col IV was that the number of Col IV-positive EVCT cells that invaded into the maternal interstitium was considerably higher than the number of fibronectin-positive EVCT cells (data not shown). Meanwhile, Col IV was also found in the basement membranes of villi where fibronectin was absent.

The temporal changes of immunoreactive fibronectin and Col IV in EVCT cells were also analogous. Moderate signals could be observed as early as weeks 3-4 of gestation with signal intensity (Fig. $1 \mathrm{a}$,d) and the number of positive EVCT cells peaked during weeks 5-7 of gestation (Fig. 1b,e). From week 8 of gestation, the strength of immunostaining declined, accompanied by switching of signals from intracellular to extracellular regions (Fig. 1c,f).

On the maternal side, positive staining of fibronectin and Col IV were shown in a portion of the epithelium, endothelium and a small section of stromal cells during weeks 3-4 of gestation. With the progress of gestation, 

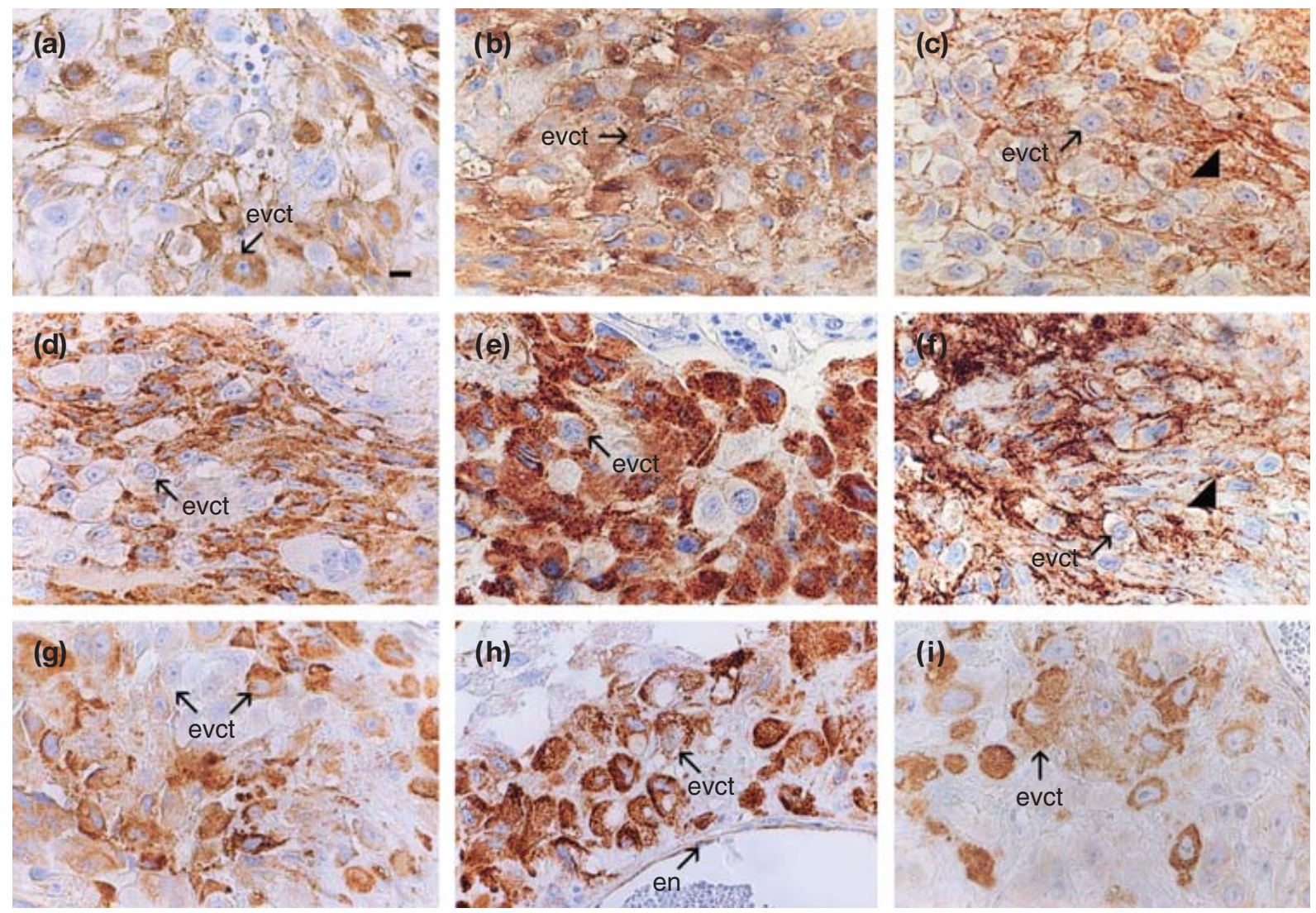

Fig. 1. Temporal alteration of fibronectin, laminin and type IV collagen (Col IV) at the maternal-fetal interface during tubal pregnancy was detected by immunohistochemistry. (a) Immunostaining of fibronectin in extravillous cytotrophoblast (EVCT) cells was relatively weak during weeks 3-4 of gestation and (b) reached a peak during weeks 5-7 of gestation. (c) Up to weeks 8-9 of gestation, numbers of positive EVCT cells and immunostaining intensity declined. Arrowhead represents filament of fibronectin. $(d-f)$ Similar changes of immunoreactive Col IV in EVCT cells during weeks 3-9 of gestation. Arrowhead represents filament of Col IV. (g) Moderate laminin immunoreactivity was observed in EVCT cells during weeks 3-4 of gestation, reached a peak during weeks 5-7 (h) and decreased during weeks 8-9 of gestation (i). EVCT cells and blood vescular endothelium are represented by evct and en, respectively. Magnification $=\times 400$ (scale bar represents $10 \mu \mathrm{m})$.

no significant changes in immunoreactive fibronectin were observed. The level of immunoreactive Col IV in the epithelium was increased by weeks 5-7 of gestation, and then declined slightly during weeks $8-9$ of gestation (data not shown). Immunoreactive laminin was exhibited in cytoplasm and extracellular regions of villous CTB, syncytiotrophoblast, column CTB and invasive EVCT cells (Fig. 1g,h), as well as the basement membranes of villi and maternal blood vessels. Along the invasive pathway, the signal intensity in trophoblasts increased from villous CTB to invasive EVCT cells. In contrast to fibronectin and Col IV, laminin was strongly stained in a number of EVCT cells that had deeply invaded maternal tissue and blood vessels.

Laminin immunoreactivity in trophoblast cells also displayed a temporal alteration. In villous CTB, strong signals were present during weeks $3-4$ of gestation and then decreased from weeks 5-7. However, in villous syncytiotrophoblast, the converse pattern was displayed. In EVCT cells, relatively feeble immunoreactivity was exhibited during weeks 3-4 of gestation (Fig. 1g). A peak was observed during weeks 5-7 of gestation, especially in those cells invading into maternal blood vessels (Fig. 1h). Immunoreactivity declined from week 8 of gestation, after which only EVCT cells that invaded to maternal blood vessels displayed positive staining (Fig. 1i).

On the maternal side of the interface, endothelial and some stromal cells displayed positive staining for laminin, whereas epithelial cells did not. During weeks 3-9 of gestation, no significant changes in laminin staining intensity were observed.

Immunoreactivity of fibronectin, laminin and Col IV as well as their spatial and temporal changes are summarized in Table 2.

Expression patterns of integrin $\alpha_{1}, \beta_{1}, \alpha_{5}$ and $\alpha_{5} \beta_{1}$ at the maternal-fetal interface during the weeks 3-9 of tubal implantation

Integrin $\alpha_{1}$ and $\beta_{1}$ were widely expressed at the maternal-fetal interface. In situ hybridization showed 

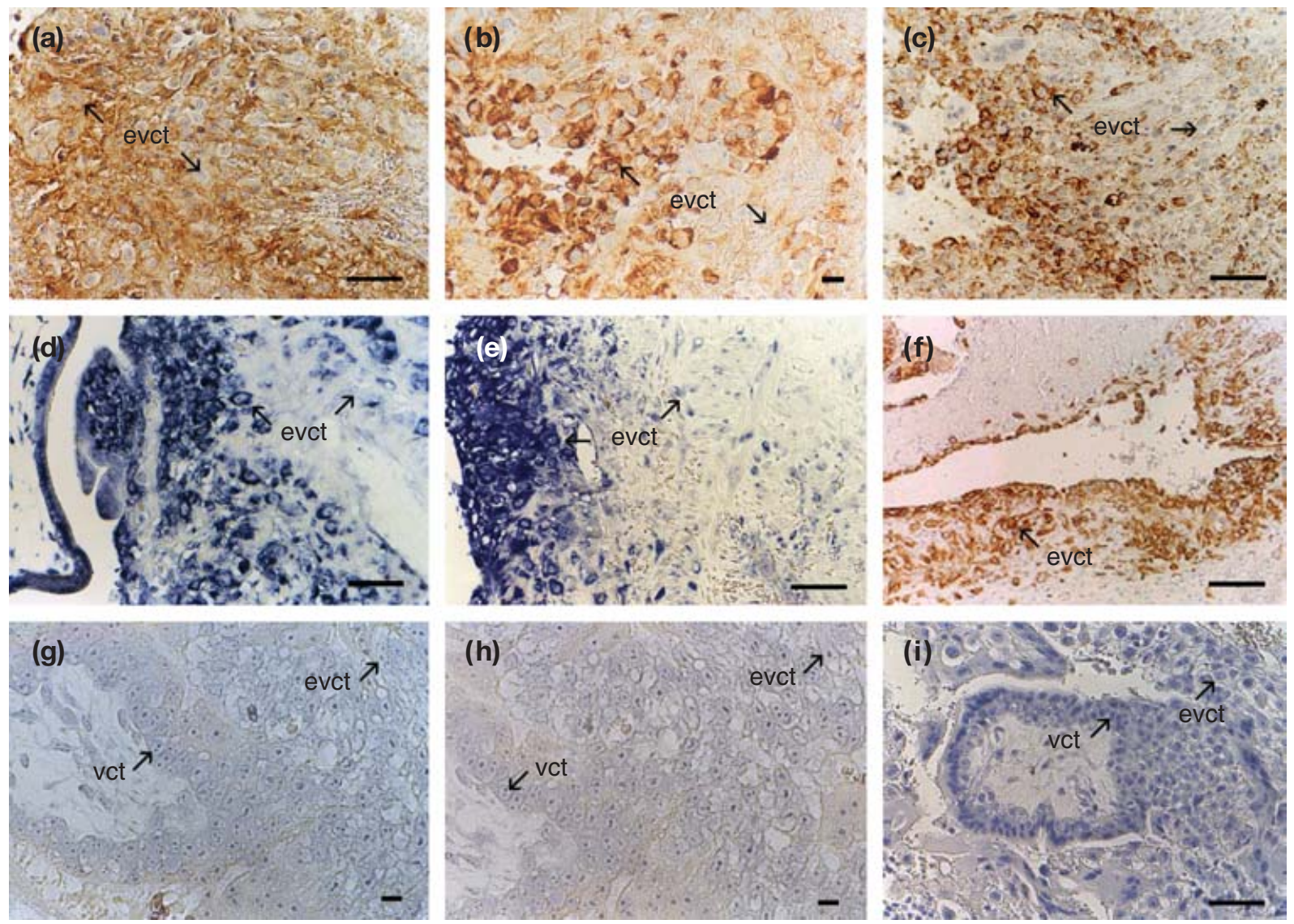

Fig. 2. Immunoreactivity of (a) fibronectin, (b) type IV collagen (Col IV) and (c) integrin $\alpha_{5} \beta_{1}$ in extravillous cytotrophoblast (EVCT) cells as well as the expression of (d) integrin $\alpha_{5}$ and (e) $\beta_{1}$ decreased with increasing penetration of maternal tissue. (f) Immunostaining of cytokeratin in EVCT cells. (g) and (h) Sense of integrin $\alpha_{5}$ and $\beta_{1}$, respectively. (i) Negative control for immunostaining. EVCT cells and villous cytotrophoblastic cells are represented by evct and vct, respectively. Magnification $=$ $\times 100(\mathrm{a}, \mathrm{c}-\mathrm{f}, \mathrm{i})($ scale bar represents $100 \mu \mathrm{m}) ; \times 200$ (b) (scale bar represents $20 \mu \mathrm{m}) ;$ and $\times 400(\mathrm{~g}$, h) (scale bar represents $10 \mu \mathrm{m})$.

Table 2. Immunostaining of extracellular matrix and integrin receptors at the maternal-fetal interface during tubal pregnancy

\begin{tabular}{lcccccc}
\hline & Fibronectin & Laminin & Col IV & Integrin $\alpha_{1}$ & Integrin $\beta_{1}$ & Integrin $\alpha_{5} \beta_{1}$ \\
\hline Villous CTB cells & - & ++ & - & + & + & + \\
ST cells & - & $+/-$ & - & $+/-$ & $(+/-)$ & $(+/-)$ \\
stromal cells & +- & $(+/-)$ & $+/-$ & $+/-$ & $(+/-)$ & $(+/-)$ \\
Proximal column & $(+/-)$ & ++ & $+/-$ & + & + & + \\
Distal column & + & ++ & + & ++ & ++ & ++ \\
Invading EVCT & ++ & +++ & +++ & +++ & +++ & ++ \\
Maternal epithelium & $+/$ & - & $+/-$ & +++ & + & ++ \\
$\quad$ endothelium & + & ++ & + & ++ & + & ++ \\
stromal cells & $(+/-)$ & $+/-$ & $(+/-)$ & $+/-$ & + & $+/-$ \\
\hline
\end{tabular}

+++ very strong staining; ++ strong staining; + positive staining; +/- positive and negative area present; $(+/-)$ negative and weakly positive area present; - negative. Col IV: type IV collagen; CTB: cytotrophoblastic cells; EVCT: extravillous cytotrophoblastic cells; ST: syncytiotrophoblast.

that mRNA of $\alpha_{1}$ and $\beta_{1}$ subunits was transcribed in villous CTB, mesenchyme and some syncytiotrophoblast and EVCT cells (Fig. 3a,e). The expression of both types of subunits was upregulated along the invasive pathway, with invasive EVCT cells displaying the highest expres- sion, but the expression of $\beta_{1}$ decreased in EVCT cells invading deeply into maternal tissues (Fig. 2e). By weeks 3-4 of gestation there was moderate expression of $\alpha_{1}$ and $\beta_{1}$ subunits in EVCT cells (Fig. 3a,e). From week 5 to week 9 of gestation, expression of the $\alpha_{1}$ subunit remained 

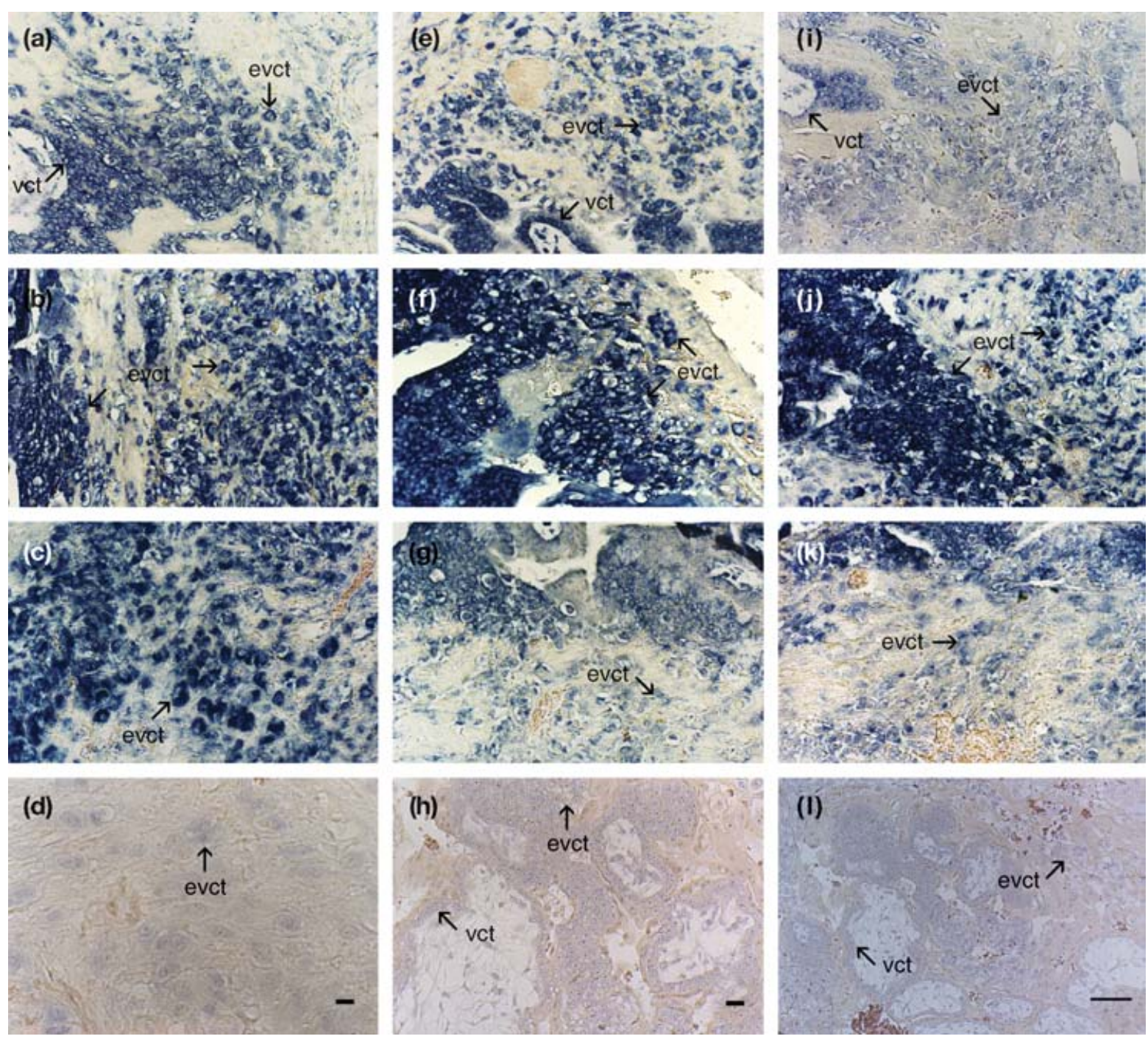

Fig. 3. Expression of integrins at the maternal-fetal interface during weeks 3-9 of tubal pregnancy was revealed by in situ hybridization. (a) Integrin $\alpha_{1}$ was moderately expressed in extravillous cytotrophoblast (EVCT) cells during weeks 3-4 of gestation. (b,c) The expression of $\alpha_{1}$ was maintained at a high level during weeks 5-9 of gestation. (d) Sense of integrin $\alpha_{1}$. (e) Moderate levels of integrin $\beta_{1}$ mRNA were expressed in EVCT cells during weeks 3-4 of gestation and (f) reached a peak during weeks $5-7$ of gestation. (g) The expression decreased slightly up to weeks 8-9 of gestation. (h) Sense of integrin $\beta_{1}$. (i) Relatively lower expression of $\alpha_{5}$ was detected in EVCT cells during weeks 3-4 of gestation. The expression increased during weeks 5-7 of gestation (j) then declined up to weeks 8-9 of gestation (k). (l) Sense for integrin $\alpha_{5}$ subunit. EVCT cells and villous cytotrophoblastic cells are represented by evct and vct, respectively. Magnification $=\times 100(\mathrm{a}-\mathrm{c}, \mathrm{e}-\mathrm{g}$, $\mathrm{i}-\mathrm{l})(\mathrm{scale}$ bar represents $100 \mu \mathrm{m}$ ); $\times 400$ (d) (scale bar represents $10 \mu \mathrm{m}$ ); and $\times 200$ (h) (scale bar represents $20 \mu \mathrm{m}$ ).

high (Fig. 3b,c). $\beta_{1}$ expression was high in weeks $5-7$ (Fig. 3f), but declined slightly during weeks 8-9 of gestation (Fig. 3g). No significant alteration of either subunit was observed from week 3 to week 9 of gestation in villous CTB.

The distribution pattern of immunoreactive $\alpha_{1}$ and $\beta_{1}$ in trophoblast cells was almost the same as that of mRNA transcription as revealed by in situ hybridization. The only difference was that only a small number of villous CTB cells exhibited positive immunostaining of $\beta_{1}$.

In the maternal interstitium, epithelial, endothelial and stromal cells expressed integrin $\alpha_{1}$ and $\beta_{1}$. The mRNA of these two types of integrin subunits transcribed at a higher level in the epithelium, and at a lower level in stromal and endothelial cells. Moreover, the mRNA level and immunoreactivity of both subunits in maternal cells showed no significant alteration during weeks 3-9 of gestation.

Integrin $\alpha_{5}$ was also extensively expressed at the maternal-fetal interface. In situ hybridization revealed its expression in villous CTB, syncytiotrophoblast and column CTB as well as in most EVCT cells (Fig. $3 \mathrm{i}$ ). The expression ascended from villous CTB to EVCT cells, but decreased in EVCT that had deeply invaded into maternal 
tissue (Fig. 2d). Villous syncytiotrophoblast exhibited lower expression. During weeks 3-4 of gestation, expression of $\alpha_{5}$ mRNA in EVCT cells was moderate (Fig. 3i), reached a peak during weeks 5-7 (Fig. 3j), and then decreased by weeks 8-9 of gestation (Fig. 3k). However, no significant alteration of its expression was observed in villous CTB cells during weeks 3-9 of gestation.

On the maternal side, expression of integrin $\alpha_{5}$ was different in epithelial, endothelial and stromal cells. The level of integrin $\alpha_{5}$ was usually high in epithelial cells, but relatively low in endothelial and stromal cells. No significant changes in the expression of integrin $\alpha_{5}$ were observed in these cells with the progress of gestation.

Integrin $\alpha_{5} \beta_{1}$ heterodimer was detected by immunohistochemistry. Positive immunoreaction was exhibited in the cell membranes and cytoplasm of villous CTB, column CTB and most invasive EVCT cells (Fig. 2c). Consistent with the mRNA expression pattern of the $\alpha_{5}$ subunit, the signal intensity of immunoreactive $\alpha_{5} \beta_{1}$ increased from villous CTB to EVCT cells that had superficially invaded maternal tissue, but decreased in those that had penetrated more deeply (Fig. 2c).

On the maternal side, immunoreactive $\alpha_{5} \beta_{1}$ was highly evident in epithelial and endothelial cells, but only slightly so in some stromal cells. The temporal alteration of immunoreactive $\alpha_{5} \beta_{1}$ was the same as that of the $\alpha_{5}$ mRNA during weeks 3-9 of gestation in both trophoblast and maternal cells.

\section{Discussion}

This study is the first to detect the spatial and temporal expression patterns of integrin $\alpha_{1}, \beta_{1}, \alpha_{5}$ and $\alpha_{5} \beta_{1}$, and their ECM ligands including fibronectin, laminin and Col IV, at the maternal-fetal interface during tubal pregnancy at both protein and mRNA levels. The results obtained by in situ hybridization were consistent with those of immunohistochemistry.

The analogue of tubal pregnancy revealed that expression of integrin $\alpha_{1}, \beta_{1}, \alpha_{5}, \alpha_{5} \beta_{1}$ and their corresponding ECM ligands by EVCT cells was moderate as early as weeks 3-4 of gestation. Previous studies indicate that fibronectin and laminin mediate cell adhesion and invasion by binding with corresponding integrin receptors (Duband, 1990; Korhonen and Virtanen, 1997). Evidence also indicates that the co-expression of integrins and ECM ligands in monkey trophoblasts play an important role in mediating the adhesion of embryos to acceptable endometrium during implantation (Blankenship et al., 1992; Fazleabas et al., 1997). The apparent early expression of integrins and their ECM ligands in trophoblasts during Fallopian pregnancy may act in a similar way. The expression of these molecules peaked during weeks 5-7 of gestation and then declined slightly by weeks 8-9. Histologically, invasion of maternal tissue by trophoblasts occurred by weeks $3-4$ of gestation when only a small number of EVCT cells were distributed on the superficial part of the Fallopian interstitium. During weeks 5-9 of gestation, large numbers of EVCT cells extensively invaded the Fallopian stroma and even blood vessels; meanwhile, villous blood vessels appeared. Another parallel study also revealed the strong expression of MMP-2, -9 and TIMP-1, -2, -3 in EVCT cells during weeks 5-9 of gestation (Bai et al., 2001). In an in vitro model, Xu et al. (2001) indicated that laminin and fibronectin could promote the migration of cultured human CTB cells with the induced expression of MMP9, TIMP-1 and TIMP-3. On the basis of these data, the present authors suggest that the ascending expression of integrins and ECM ligands might promote the invasion of the Fallopian tube by EVCT cells by auto-regulating the expression and secretion of certain types of MMPs and TIMPs through the interaction of integrins and ECM ligands. The slight reduction in the expression of integrins and ECM ligands during weeks 8-9 of gestation indicates decreased capability of trophoblast invasion. The data seem to indicate that the invasive potential of trophoblast cells is strongest during the second month of tubal implantation.

The spatial changes of integrins and ECM ligands at the maternal-fetal interface were also revealed in the present study. Along the invasive pathway, the expression of integrin $\alpha_{1}, \beta_{1}$ as well as laminin and Col IV increased. Integrin $\alpha_{5} \beta_{1}$ and fibronectin displayed an analogous ascending pattern. Evidence from normal intrauterine pregnancy indicates that integrin $\alpha_{1} \beta_{1}$ and $\alpha_{5} \beta_{1}$ were upregulated in differentiating and invasive CTBs (Damsky et al., 1992, 1994). Using an in vitro invasion model, Damsky et al. (1994) further demonstrated that interactions involving laminin or Col IV and their $\alpha_{1} \beta_{1}$ integrin receptor promoted invasion by CTB cells, whereas interactions between fibronectin and integrin $\alpha_{5} \beta_{1}$ inhibited invasion. Damsky et al. (1994) suggested that integrin switching observed during differentiation had significant functional consequences for CTB invasion, and that counterbalanced invasion-accelerating and invasion-restraining adhesion mechanisms were present in differentiating CTBs. In the present study, correspondingly ascending expression of fibronectin, laminin and Col IV as well as their integrin receptors $\alpha_{1} \beta_{1}$ and $\alpha_{5} \beta_{1}$ was observed in differentiating EVCT cells. This might also indicate their counterbalanced contribution to the invasion of the Fallopian tube by trophoblast cells during tubal implantation. Meanwhile, it was found that the expression of $\beta_{1}$ and $\alpha_{5} \beta_{1}$, as well as fibronectin and Col IV decreased in EVCT cells that invaded into deeper parts of the maternal interstitium compared with those located at the distal column and shallower invasion sites. Evidence from cultured explant villi from normal pregnancies revealed the same alteration and consequently indicates the same controlled invasion mechanism (Vicovac et al., 1995; Aplin et al., 2000). In tubal pregnancy, the embryo implants in the Fallopian tube rather than the uterine endometrium. There has 
been evidence indicating that the Fallopian stroma is devoid of a decidual reaction during tubal implantation (Vassiliadou and Bulmer, 1998), which would result in lack of inhibition of EVCT cells by the maternal decidua, thus leading to excessive invasion of trophoblasts into maternal tissue. However, the observation that the expression of integrins and ECM ligands in EVCT cells decreased with their level of penetration of the Fallopian endometrium implies the presence of a regulatory mechanism governing trophoblast invasion in tubal pregnancy. There is probably a gene programme to determine the direction and extent of trophoblast differentiation. Environmental factors may activate or inhibit the commencement of the programme to regulate cell differentiation subtly.

One interesting observation was that EVCT cells that distributed around or inside maternal blood vessels often possessed much stronger laminin immunoreactivity compared with those located in other maternal tissues. Moreover, with the progress of gestation, especially during weeks 8-9, only this group of trophoblast cells showed strong staining for laminin. This finding indicates that these EVCT cells were different from other invading trophoblast cells. The degradation and reconstruction of maternal blood vessels at the maternalfetal interface during embryo implantation is critical for the establishment of circulation between the mother and conceptus. There is evidence to show that laminin can induce the secretion of proteases, that is MMP2, and therefore enhance the remodelling of the ECM (Yudoh et al., 1995). EVCT cells located around maternal blood vessels during tubal implantation displayed strong expression of MMP-2 (Bai et al., 2001). Hence, it is likely that laminin produced by EVCT cells would not only facilitate their adhesion to maternal blood vessels, but also accelerate their invasion of the maternal blood vessels by inducing the expression and secretion of MMP2 in an autocrine manner to degrade ECM ligands in the vascular basement membrane. These EVCT cells may possess stronger adhesive and invasive potential relative to other trophoblast cells invading the maternal interstitium.

Another observation was that with the progress of gestation, signals of fibronectin and Col IV in EVCT cells switched from the cytoplasm to extracellular regions. By weeks 8-9 of gestation, many EVCT cells had invaded deep into the maternal interstitium and were surrounded by a positive filament deposit with negative staining in the cytoplasm. This distribution pattern indicates that the synthesis of ECM ligands by EVCT is performed during this special stage, and the secretion of ECM ligands in the extracellular region might not only facilitate the counterbalanced adhesion to and invasion of maternal tissues by EVCT, but also contribute to the remodelling of maternal tissue during implantation.

On the maternal side, certain integrins and ECM ligands were found to be produced by epithelial, endothelial and stromal cells. These ECM proteins might contribute significantly to the remodelling of maternal tissues during implantation. On the other hand, expression of TIMP-1, -2, -3 by these cells was low (S. X. Bai, Y. L. Wang, L. Qin, R. Herva, S. H. Ji, Z. J. Xiao and Y. S. Piao, unpublished). An autocrine regulation of TIMPs by the interaction of integrin and ECM ligands is probably also present in Fallopian tube cells, and this may contribute to controlled trophoblast invasion. Meanwhile, integrins may mediate the adhesion of EVCT cells surrounded by ECM ligands to the maternal interstitium. In addition, the interaction between maternal ECM ligands, integrins and trophoblast cells may further induce the expression and secretion of MMPs-TIMPs in the maternal interstitium and facilitate the extensive migration of EVCT in maternal Fallopian tissue.

This study determined that there are many similarities in the distribution of integrins and ECM ligands between tubal and normal pregnancy. For example, fibronectin and Col IV were detected in column CTB cells and some invasive EVCT cells, but were not observed in villous trophoblast cells. Laminin was detected in both villous CTB and many EVCT cells, and its immunoreactivity increased from villous CTB to EVCT cells. Integrin $\alpha_{1}$, $\beta_{1}, \alpha_{5}$ as well as $\alpha_{5} \beta_{1}$ were widely expressed in EVCT cells. These observations are consistent with the available data on normal uterine implantation in humans (Earl et al., 1990; Autio-Harmainen et al., 1991; Damsky et al., 1992, 1994; Burrows et al., 1993; Huppertz et al., 1996; Korhonen and Virtanen, 1997, 2001). Consequently, the present data indicate that tubal pregnancy may provide an ideal analogue for investigating the mechanisms of trophoblast adhesion and invasion during human embryo implantation. Such an analogue is particularly useful as specimens from weeks 3-4 of gestation are rarely available from normal pregnancies but can be obtained from tubal pregnancies.

In summary, study of the spatial and temporal expression pattern of various integrins and their corresponding ECM ligands at the maternal-fetal interface of tubal pregnancy provides better understanding of the mechanisms involved in trophoblast adhesion and invasion during human embryo implantation, especially at the very early stages of gestation.

This work was supported by the Special Funds for Major State Basic Research Project (No. G1999055903) and the Major Research Projects of the CAS Knowledge Innovation Program (KSCX-2-SW-201). The authors thank L. Z. Zhuang and R. Moorhouse for their helpful comments on the manuscript.

\section{References}

Aplin JD, Haigh T, Jones CJ, Church HJ and Vicovac L (1999) Development of cytotrophoblast columns from explanted first-trimester human placental villi: role of fibronectin and integrin alpha5beta1 Biology of Reproduction 60 828-838 
Aplin JD, Haigh T, Lacey H, Chen CP and Jones CJ (2000) Tissue interactions in the control of trophoblast invasion Journal of Reproduction and Fertility Supplement $\mathbf{5 5}$ 57-64

Autio-Harmainen H, Sandberg M, Pihlajaniemi T and Vuorio E (1991) Synthesis of laminin and type IV collagen by trophoblastic cells and fibroblast stromal cells in the early human placenta Laboratory Investigation 64 483-491

Bai SX, Wang YL, Qin L, Herva R, Ji SH, Xiao ZJ and Piao YS (2001) Temporal and spatial expression of MMP-2, -9 and -14 at the fetalmaternal interface during tubal pregnancy of humans Developmental and Reproductive Biology Supplement 1096 (Abstract)

Blankenship TN, Enders AC and King BF (1992) Distribution of laminin, type IV collagen and fibronectin in the cell columns and trophoblastic shell of early macaque placentas Cell and Tissue Research 270 241-248

Braissant O and Wahli W (1998) A simplified in situ hybridization protocol using non-radioactively labeled probes to detect abundant and rare mRNA on tissue sections Biochemica 10-16

Burrows TD, King A and Loke YW (1993) Expression of integrins by human trophoblast and differential adhesion to laminin and fibronectin Human Reproduction 8 475-484

Critchley DR (2000) Focal adhesions - the cytoskeletal connection Current Opinion in Cell Biology 12 133-139

Damsky CH, Fitzgerald ML and Fisher SJ (1992) Distribution of extracellular matrix components and adhesion receptors are intricately modulated during first trimester cytotrophoblast differentiation along the invasive pathway in vivo. Journal of Clinical Investigation 89 210-222

Damsky CH, Librach C, Lim KH, Fitzgerald ML, McMaster MT, Janatpour M, Zhou Y, Logan SK and Fisher SJ (1994) Integrin switching regulates normal trophoblast invasion Development 120 3657-3666

Duband JL (1990) Extracellular matrix and embryonal morphogenesis: role of fibronectin in cell migration Reproduction, Nutrition, Development 30 379-395

Earl U, Wells M and Bulmer JN (1986) Immunohistochemical characterization of trophoblast antigens and secretory products in ectopic tubal pregnancy International Journal of Gynecological Pathology 5 132-142

Earl U, Estlin C and Bulmer JN (1990) Fibronectin and laminin in the early human placenta Placenta 11 223-231

Fazleabas AT, Bell SC, Fleming S, Sun J and Lessey BA (1997) Distribution of integrins and the extracellular matrix proteins in the baboon endometrium during the menstrual cycle and early pregnancy Biology of Reproduction $\mathbf{5 6}$ 348-356

Fisher SJ and Damsky CH (1993) Human cytotrophoblast invasion Seminars in Cell Biology 4 183-188

Floridon C, Nielsen O, Holund B, Sunde L, Westergaard JG, Thomsen SG and Teisner B (1999) Localization and significance of urokinase plasminogen activator and its receptor in placenta tissue from intrauterine, ectopic and molar pregnancies Placenta 20 711-721

Furuhashi N, Kimura H, Nagae H, Yajima A, Kimura C and Saito T (1994) Serum collagen IV and laminin levels in pre-eclampsia Gynecologic and Obstetric Investigation 37 250-253

Gardner H, Kreidberg J, Koteliansky V and Jaenisch R (1996) Deletion of integrin alpha 1 by homologous recombination permits normal murine development but gives rise to a specific deficit in cell adhesion Developmental Biology 175 301-313

Huppertz B, Kertschanska S, Frank HG, Gaus G, Funayama H and Kaufmann P (1996) Extracellular matrix components of the placental extravillous trophoblast: immunocytochemistry and ultrastructural distribution Histochemistry and Cell Biology 106 291-301

Huppertz B, Kertschanska S, Demir AY, Frank HG and Kaufmann P (1998) Immunohistochemistry of matrix metalloproteinases (MMP), their substrates, and their inhibitors (TIMP) during trophoblast invasion in the human placenta Cell and Tissue Research 291 133-148

Kaplan CG (1999) Embryonic pathology of the placenta. In Pathology of the Placenta 2nd Edn pp 89-104 Eds SH Lewis and E Perrin. Churchill Livingstone Press, New York

Korhonen M and Virtanen I (1997) The distribution of laminins and fibronectins is modulated during extravillous trophoblastic cell differentiation and decidual cell response to invasion in the human placenta Journal of Histochemistry and Cytochemistry 45 569-581
Korhonen M and Virtanen I (2001) Immunohistochemical localization of laminin and fibronectin isoforms in human placental villi Journal of Histochemistry and Cytochemistry 49 313-322

Kucera E, Tangl S, Klem I, Konig F, Grossschmidt K, Kainz C and Sliutz G (2000) Immunohistochemical expression of matrix metalloproteinases 1 and 2 (MMP-1 and MMP-2) and tissue inhibitor of metalloproteinase 2 (TIMP) in ruptured and non-ruptured tubal ectopic pregnancies Wiener klinische Wochenschrift 12 749-753

Lin CQ and Bissell MJ (1993) Multi-faceted regulation of cell differentiation by extracellular matrix FASEB Journal 7 737-743

Rubin K, Gullberg D, Tomasini-Johansson B, Reed RK, Ryden C and Borg TK (1996) Molecular recognition of the extracellular matrix by cell surface receptors. In Extracellular Matrix Vol 2 pp 269-272 Ed. Wayne D Comper. Harwood Academic, Netherlands

Schaller MD, Borgman CA, Cobb BS, Vines RR, Reynolds AB and Parsons JT (1992) pp125FAK, a structurally unique protein-tyrosine kinase associated with focal adhesions Proceedings National Academy of Sciences USA 89 5192-5196

Schoenwaelder SM and Burridge K (1999) Bidirectional signaling between the cytoskeleton and integrins Current Opinion in Cell Biology 11274 286

Smyth N, Vatansever HS, Meyer M, Frie C, Paulsson M and Edgar D (1998) The targeted deletion of the LAMC1 gene Annals of the New York Academy of Sciences 857 283-286

Stephens LE, Sutherland AE, Klimanskaya IV, Andrieux A, Meneses J, Pedersen RA and Damsky CH (1995) Deletion of beta 1 integrins in mice results in inner cell mass failure and peri-implantation lethality Genes and Development 9 1883-1895

Tarone G, Hirsch E, Brancaccio M et al. (2000) Integrin function and regulation in development International Journal of Developmental Biology 44 725-731

Van der Rest M and Garrone R (1991) Collagen family of proteins FASEB Journal 5 2814-2823

Vassiliadou N and Bulmer JN (1998) Characterization of tubal and decidual leukocyte populations in ectopic pregnancy: evidence that endometrial granulated lymphocytes are absent from the tubal implantation site Fertility and Sterility $69760-767$

Vicovac L, Jones CJ and Aplin JD (1995) Trophoblast differentiation during formation of anchoring villi in a model of the early human placenta in vitro. Placenta 16 41-56

Virtanen I, Gullberg D, Rissanen J, Kivilaakso E, Kiviluoto T, Laitinen LA, Lehto VP and Ekblom P (2000) Laminin alpha1-chain shows a restricted distribution in epithelial basement membranes of fetal and adult human tissues Experimental Cell Research 257 298-309

Wallner EI, Yang Q, Peterson DR, Wada J and Kanwar YS (1998) Relevance of extracellular matrix, its receptors, and cell adhesion molecules in mammalian nephrogenesis American Journal of Physiology 275 467477

Watt FM and Hodivala KJ (1994) Cell adhesion. Fibronectin and integrin knockouts come unstuck Current Biology 4 270-272

Xu P, Wang YI, Piao YS, Bai SX, Xiao ZJ, Luo SY and Zhuang LZ (2001) Effects of matrix proteins on the expression of matrix metalloproteinase$2,-9$ and -14 and tissue inhibitor of metalloproteinases in human cytotrophoblast cells during the first trimester Biology of Reproduction 65 240-246

Yang JT, Rayburn H and Hynes RO (1993) Embryonic mesodermal defects in alpha 5 integrin-deficient mice Development 1191093 1105

Yudoh K, Matsui H, Kanamori M, Ohmori K and Tsuji H (1995) Tumor cell attachment to laminin promotes degradation of the extracellular matrix and cell migration in high-metastatic clone cells of RCT sarcoma in vitro. Japanese Journal of Cancer Research 86 685-690

Received 11 March 2003.

First decision 19 May 2003.

Revised manuscript received 9 June 2003.

Accepted 12 June 2003. 